\title{
Amino Monolayer Modified Nanowire Array for Trinitrotoluene Detection
}

\author{
Xingqi Liu, ${ }^{1,2}$ Shixing Chen, ${ }^{2}$ Hui Wang, ${ }^{2}$ Anrao Gao, ${ }^{2}$ Yuelin Wang, ${ }^{2}$ Tie Li, ${ }^{2 *}$ \\ Hongpeng Zhang, ${ }^{1}$ Zhiping Huang, ${ }^{1}$ and Zhenxing Cheng ${ }^{1 * *}$ \\ ${ }^{1}$ Department of Chemical Defence, Institute of NBC Defence, Beijing 102205, China \\ ${ }^{2}$ State Key Laboratory of Functional Materials for Informatics, Shanghai Institute of Microsystem \\ and Information Technology, Chinese Academy of Sciences, Shanghai 200050, China
}

(Received May 31, 2018; accepted August 10, 2018)

Keywords: silicon nanowire array, detection, trinitrotoluene, silanization

In this paper, we reported a homemade silicon chemical sensor of arranged silicon nanowires for the detection of 2,4,6-trinitrotoluene (TNT) explosive, where the key sensor structure surface has been successfully modified into an amino monolayer with (3-aminopropyl)dimethylethoxysilane (APDMES) through molecular oligomerization in a self-assembled manner. The sensor can easily and rapidly detect both TNT dissolved in the liquid phase from 5 $\mathrm{fM}$ to $50 \mathrm{pM}$ and TNT vapor in air approximately at about $1 \mathrm{ppb}$. More importantly, the sensors showed a rather lower response sensitivity to several TNT analogs than to TNT.

\section{Introduction}

In recent years, explosive terrorist attacks have become one of the major threats to global security. Typically, 2,4,6-trinitrotoluene (TNT), a widely used explosive, leads to both security and environmental concerns, so the detection of TNT has attracted extensive attention.

Currently, besides off-site chemical analysis, there also exist various analytical techniques for the analysis of explosives in situ, such as gas chromatography/mass spectrometry (GC/MS), ${ }^{(1,2)}$ fluorescence, ${ }^{(3-5)}$ Raman spectrometry, ${ }^{(6,7)}$ and ion mobility spectrometry. ${ }^{(8)}$ All of these methods exhibit high capability, but are often bulky, expensive, and time-consuming. Because of these limitations, there is still an urgent need for portable, low-cost, and low-powerconsumption devices for the detection of explosives in the environment and for homeland security.

The development of nanoelectromechanical systems (NEMS) provides unprecedented opportunities for developing ideal trace explosive sensors that satisfy all the requirements outlined above. ${ }^{(9-11)}$ NEMS chips not only have the advantages of small size and low power consumption but also achieve high sensitivity, which cannot be observed with macroscale materials. In particular, the silicon nanowire array has attracted a great deal of attention because of its exquisite sensitivity and fast response to the surrounding environment. ${ }^{(12-14)}$

\footnotetext{
*Corresponding author: e-mail: tli@mail.sim.ac.cn

** Corresponding author: e-mail: chengzx2018@sina.com https://doi.org/10.18494/SAM.2018.2015
} 
Thus far, several groups have reported the use of silicon nanowire devices for the detection of TNT. ${ }^{(15-19)}$ Patolsky's group traced TNT using amino-terminated silicon nanowire devices, 16 of which could detect TNT with a limit of femtomolar level. After a single-step chemical modification, they transferred amine groups to the silicon nanowire surface using (3-aminopropyl)-triethoxysilane (APTES), which could selectively bond to TNT to form chargetransfer complexes.

However, APTES rarely formed a true self-assembled monolayer (SAM) on the surface. As shown in Fig. 1, both the head and tail groups of APTES were oriented towards the surface, resulting in large disorder layers. ${ }^{(20,21)}$ Herein, we report a new method of generating a true monolayer on a silicon nanowire sensor without the formation of oligomerization structures on the surface using (3-aminopropyl)-dimethylethoxysilane (APDMES). APDMES is a special alkoxydimethylsilane with only one alkoxy group that forms one $\mathrm{Si}-\mathrm{O}-\mathrm{Si}$ bond with the silicon nanowire surface. The reaction on the two alkoxy groups prevents the amino group from bonding with the silicon nanowire surface. Therefore, oligomerization cannot occur and an SAM inevitably appears.

Notably, as shown in Fig. 2, TNT molecules are recognized by the acid-base interaction between the amino groups and the polarized TNT nitro groups. By using this amine-decorated nanowire sensor, we achieved the sensitive and selective detection of TNT, which induced charge transfer between the nitro group of the explosive and the silicon nanowire sensor surface.

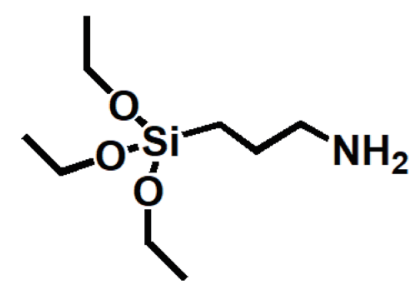

(a)

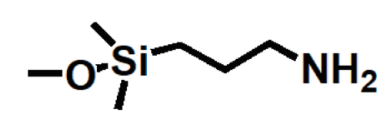

(b)

Fig. 1. Molecular structures of (a) APTES and (b) APDMES.

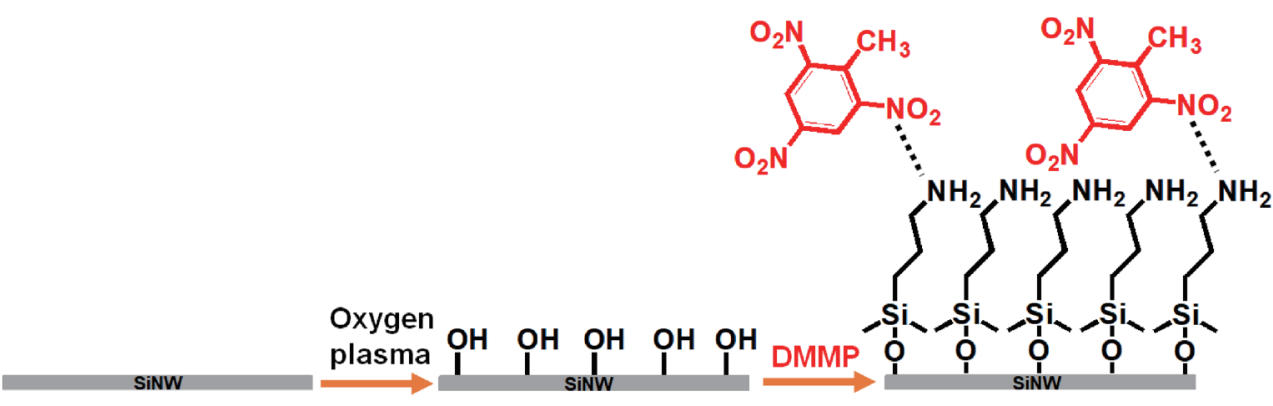

Fig. 2. (Color online) Schematic representation of surface functionalization of silicon nanowire and molecular interaction. 


\section{Materials and Methods}

\subsection{Reagents and chemicals}

APDMES was purchased from J\&K Chemical Ltd. (Beijing, China). Dimethyl sulfoxide (DMSO) was obtained from Sigma-Aldrich Inc. (St. Louis, MO, USA). TNT was obtained from the Institute of NBC Defence (Beijing, China). 1,3-Dinitrobenzene, 4-nitrophenol, and 2-nitrochlorobenzene were obtained from Beijing Beihua Fine Chemical Ltd. (Beijing, China). Ethanol was purchased from Hanyin Chemical Ltd. (Hanyin, China). The 4-inch (111) siliconon-insulator (SOI) wafer was acquired from Xinggui Commercial and Trading Co., Ltd. (Shanghai, China).

\subsection{Fabrication of silicon nanowire sensor}

The arranged silicon nanowires were designed and fabricated on (111) SOI wafers. ${ }^{(22)}$ By controllable silicon-anisotropy etching and self-limiting oxidation, we can generate silicon nanowire arrays at the top center of wall arrays, nearly with the same dimensions. As shown in Fig. 3, the arranged silicon nanowire arrays were successfully manufactured containing hundreds of same-size silicon nanowires.

\subsection{Surface functionalization of silicon nanowire sensor}

Generally, the sensors with arranged silicon nanowires were pretreated with oxygen plasma for 15 min to generate $-\mathrm{OH}$ on the $\mathrm{SiO}_{2}$ surface, which was verified by contact angle measurement on silicon wafers with water droplets since the silicon nanowire sensor device was very small. As indicated in Fig. 4, the contact angle dramatically decreased because of the hydrophilicity of hydroxyl groups after silicon wafers were treated with oxygen plasma.

Then, the sensors were treated with ethanol solution containing 2\% (v/v) APDMES at room temperature for $2 \mathrm{~h}$ to form a single layer of APDMES by $\mathrm{Si}-\mathrm{O}-\mathrm{Si}$ covalent bonding on the sensing nanowire surface. The unbound APDMES was washed off with ethanol and the sensors were cleaned with nitrogen and finally dried at $110^{\circ} \mathrm{C}$ for $15 \mathrm{~min}$.

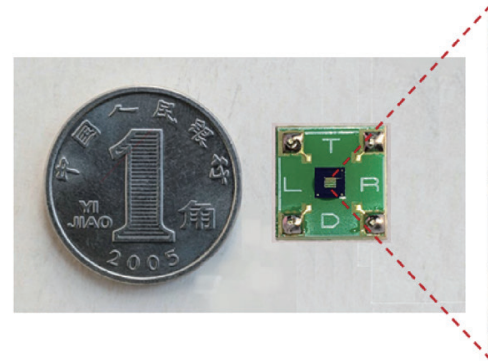

(a)

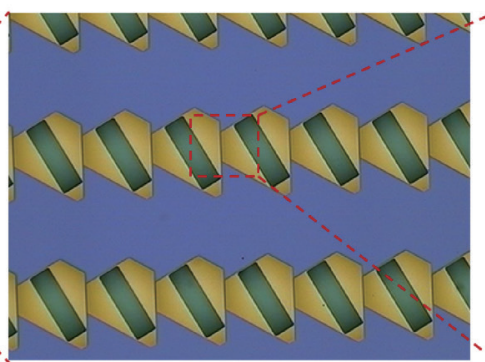

(b)

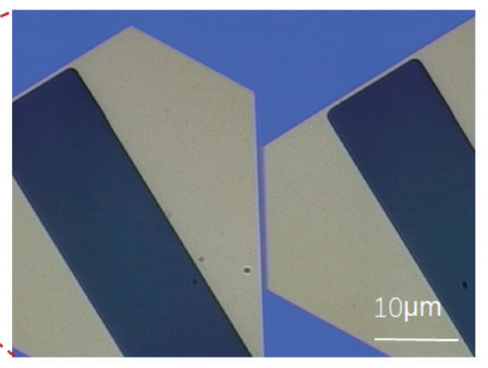

(c)

Fig. 3. (Color online) (a) Photograph of silicon nanowire array sensor. (b) Arranged silicon nanowires. (c) Image of single nanowire. 


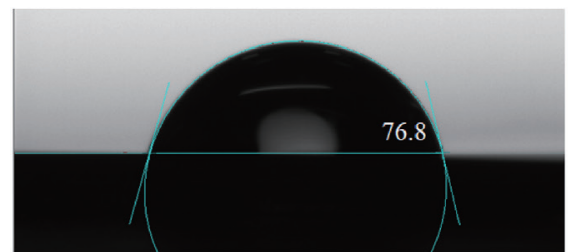

(a)

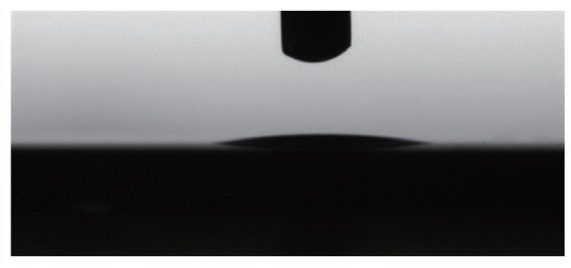

(b)

Fig. 4. (Color online) Contact angle measurements on silicon wafers by water droplet shape method for (a) bare silicon wafers and (b) silicon wafers treated with oxygen plasma.

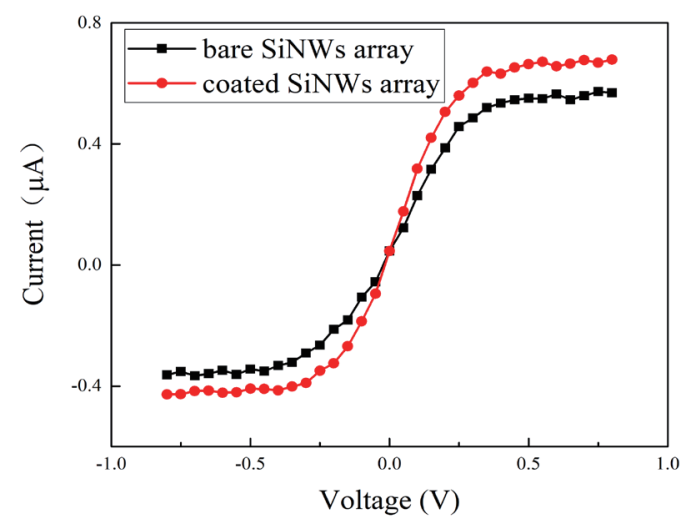

Fig. 5. (Color online) $I-V$ curve of bare silicon nanowire array (black curve) and silicon nanowire array functionalized with APDMES (red curve).

\section{Results and Discussion}

Electrical measurements in all sensing experiments were carried out using a Keithley 4200 semiconductor. As shown in Fig. 5, the current of silicon nanowire sensors slightly increased after APDMES modification, suggesting that hydroxyl groups led to an increase in negative charge on the silicon nanowire surface.

Aqueous solutions, i.e., deionized (DI) water containing 0.1\% DMSO, with TNT at concentrations ranging from $5 \mathrm{fM}$ to $50 \mathrm{pM}$ were successively added to the sensor chip device. Figure 6(a) shows the results of real-time detection of TNT with a detection limit of sub-fM level. We can observe that the current decreased when the TNT solutions were added to the chips. The inset in Fig. 6(a) indicates a very quick response to $5 \mathrm{fM}$ TNT solutions. As shown in Fig. 6(b), the conductance response of the sensor is clearly related to the concentration of TNT.

The specific detection of TNT was compared with such different nitrobenzene analogs such as 4-nitrophenol, 1,3-dinitrobenzene, and 2-nitrochlorobenzene, employing the silicon nanowire array sensors. Figure 7 shows the response of APDMES-functionalized silicon nanowire devices towards $5 \mathrm{pM}$ solutions of TNT and its analogs. It is obvious that amine-coated silicon nanowire array sensors show a strong selectivity to TNT over these analogs. 


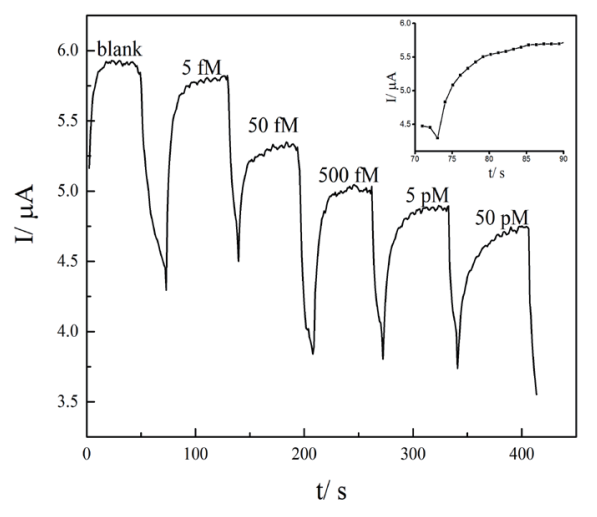

(a)

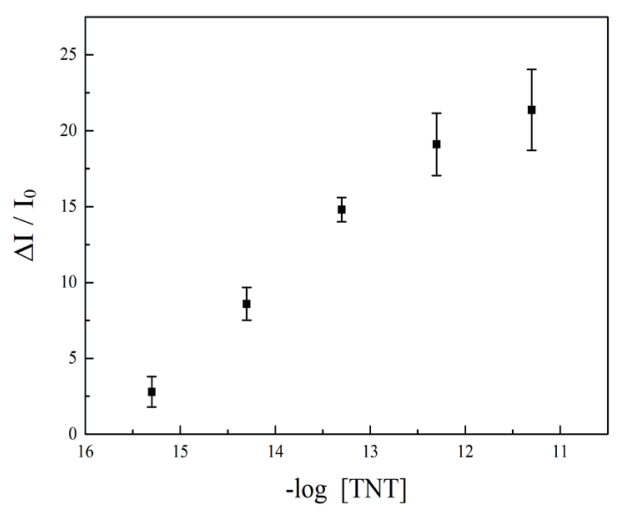

(b)

Fig. 6. (a) Measurement of TNT at concentrations of $5 \mathrm{fM}, 50 \mathrm{fM}, 500 \mathrm{fM}, 5 \mathrm{pM}$, and $50 \mathrm{pM}$. (b) Relative current change $\left(\Delta I / I_{0}\right)$ versus TNT concentration (on a logarithmic scale). The inset presents a quick response to $5 \mathrm{fM}$ TNT.

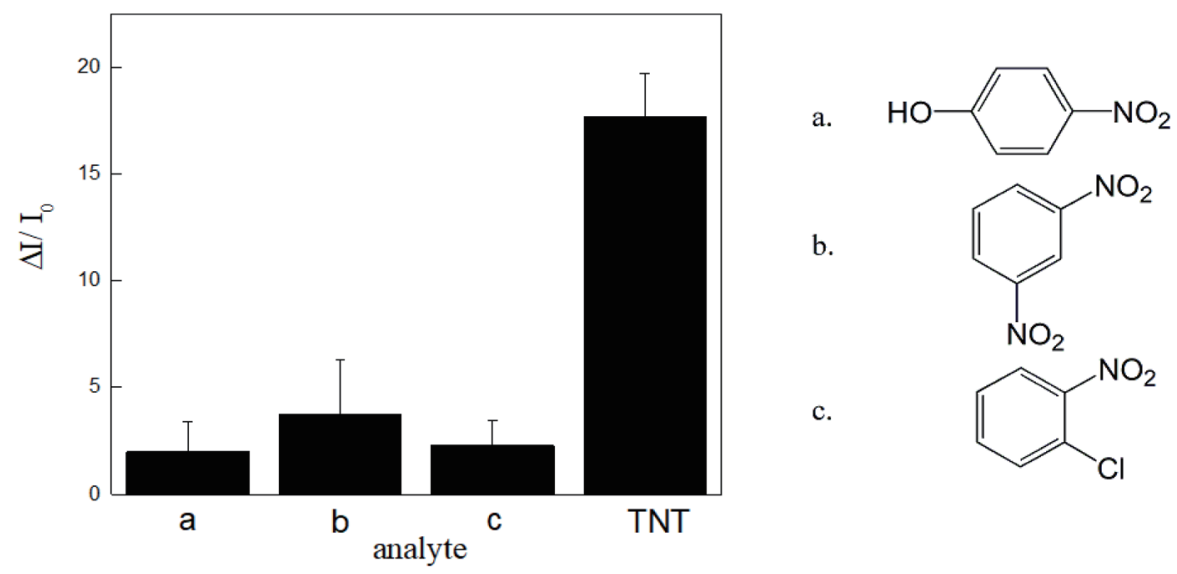

Fig. 7. (Left) Response of APDMES-functionalized silicon nanowire array device to 5 pM solutions of (a) 4-nitrophenol, (b) 1,3-dinitrobenzene, and (c) 2-nitrochlorobenzene and TNT. (Right) Molecular analogs used in this study.

Importantly, we also tested our silicon nanowire sensor to sense TNT vapor directly. The vapor pressure at ambient temperature is extremely low (approximately $1 \mathrm{ppb}$ at $20^{\circ} \mathrm{C}$ ), which means that it is difficult to trace TNT vapor molecules. As shown in Fig. 8, the response of our silicon nanowire sensor to $1 \mathrm{ppb}$ TNT could reach 30\%, indicating that our sensor can sensitively detect TNT vapor below the room-temperature saturated vapor pressure. The inset section shows that the response of a bare silicon nanowire sensor is nearly negligible, but the current markedly decreases after coating with APDMES.

The stability of the novel silicon nanowire array sensors was evaluated by comparing the TNT detection signals obtained on different dates but using the same sensor. With a typical result shown in Fig. 9, the tested sensor response to $1 \mathrm{ppb}$ TNT vapor just showed a slight attenuation one month later. 


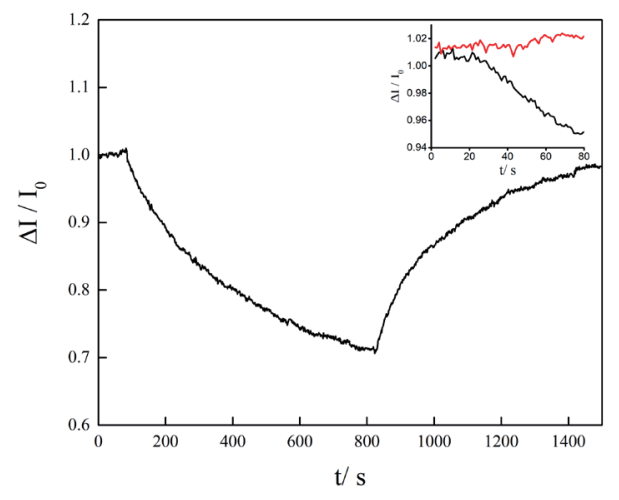

Fig. 8. (Color online) Response of sensor upon TNT vapor detection (about $1 \mathrm{ppb}$ ). Inset: bare silicon nanowire array does not respond to TNT vapor (red curve). The same device coated with amine showed clear responses to TNT (black curve). Currents are normalized to $I_{0}$ when exposed to clean air (no TNT).

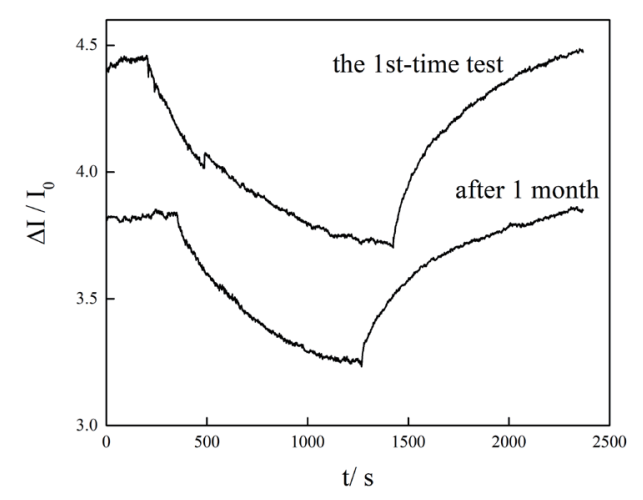

Fig. 9. Comparison of sensor response to $1 \mathrm{ppb}$ TNT vapor between the first time and 1 month later.

\section{Conclusions}

We proposed a new surface modification strategy to form a true monolayer on a silicon nanowire array with APDMES instead of APTES, avoiding oligomerization structures. We generated amine groups on a nanowire sensor and could selectively and sensitively detect TNT. We briefly concluded that the mechanism of molecular recognition was an acid-based interaction between the amino-terminated nanowire surface and the polarized TNT nitro groups. ${ }^{(23,24)}$ The binding of electron-deficient TNT molecules to the amino groups on the silicon nanowire array sensor surface resulted in the formation of charge-transfer complexes, which was called Meisenheimer complexes, leading to the current decrease of the silicon nanowire sensor. This current decrease phenomenon occurred in both aqueous solutions and vapor TNT detection.

Since silicon nanowire array sensors have gained considerable attention, our surface modification method will help the silicon nanowire sensor to be a better platform for the ultrasensitive, electrical detection of biological and chemical species. ${ }^{(25,26)}$ Thus, we hope to create, in the near future, a universal platform for the simultaneous detection of a larger spectrum of explosive chemical agents, each selectively identified by the specific electrical signal pattern measured by the silicon-sensor array.

\section{Acknowledgments}

We appreciate the financial support from the National Key Research and Development Program of China (Nos. 2017YFB0405400 and 2017YFA0207100), the Project of National Natural Science Foundation of China (Nos. 91323304, 61327811, and 91623106), and the Project for Shanghai Outstanding Academic Leaders (No. 15XD1504300). 


\section{References}

1 D. M. Gao, Z. Y. Wang, B. H. Liu, L. Ni, M. H. Wu, and Z. P. Zhang: Anal. Chem. 80 (2008) 8545. https://doi. org/10.1021/ac504667t

2 G. Dhingra, P. Bansal, N. Dhingra, S. Rani, and A. K. Malik: J. Sep. Sci. 41 (2018) 639. https://doi.org/10.1002/ jssc

3 Y. X. Gao, W. Xu, D. F. Zhu, L. Chen, Y. Y. Fu, Q. G. He, H. M. Cao, and J. G. Cheng: J. Mater. Chem. A 3 (2015) 4820. https://doi.org/10.1039/c4ta05704j

4 L. Chen, Y. X. Gao, Y. R. Wang, C. He, D. F. Zhu, Q. G. He, H. M. Cao, and J. G. Chengt: ACS Appl. Mater. Interfaces 6 (2014) 8817. https://doi.org/10.1021/am501543f

5 D. M. Gao, Z. Y. Wang, B. H. Liu, L. Ni, M. H. Wu, and Z. P. Zhang: Anal. Chem. 80 (2008) 8545. https://doi. org/10.1021/ac8014356

6 A. Chou, E. Jaatinen, R. Buividas, G. Seniutinas, S. Juodkazis, E. L. Izake, and P. M. Fredericks: Nanoscale 4 (2012) 7419. https://doi.org/10.1039/c2nr32409a

7 T. Liyanage, A. Rael, S. Shaffer, S. Zaidi, J. V. Goodpaster, and R. Sardar: Analyst 143 (2018) 2012. https://doi. org/10.1039/c8an00008e

8 S. S. Cheng, J. Dou, W. G. Wang, C. Chen, L. Hua, Q. H. Zhou, K. Y. Hou, J. H. Li, and H. Y. Li: Anal. Chem. 85 (2013) 319. https://doi.org/10.1021/ac302836f

9 L. Senesac and T. G. Thundat: Mater. Today 11 (2008) 28. https://doi.org/10.1016/S1369-7021(08)70017-8

10 G. Korotcenkov: Nanocomposites in Electrochemical Sensors: In Handbook of Gas Sensor Materials: Properties, Advantages and Shortcomings for Applications Vol. 2: New Trends and Technologies (Springer New York, NY, 2014) p. 223.

11 N. Lu, A. Gao, H. Zhou, Y. Wang, X. Yang, Y. Wang, and T. Li: Progress in Silicon Nanowire-Based FieldEffect Transistor Biosensors for Label-Free Detection of DNA (2016) Vol. 34.

12 N. Shehada, G. Bronstrup, K. Funka, S. Christiansen, M. Leja, and H. Haick: Nano Lett. 15 (2015) 1288. https://doi.org/10.1021/n1504482t

13 Y. Jin, A. R. Gao, Q. H. Jin, T. Li, Y. L. Wang, and J. L. Zhao: Nanotechnology 29 (2018). https://doi. org/10.1088/1361-6528/aaa6ec

14 R. Ermanok, O. Assad, K. Zigelboim, B. Wang, and H. Haick: ACS Appl. Mater. Interfaces 5 (2013) 11172. https://doi.org/10.1021/am403421g

15 L. J. Guo, Z. Yang, and X. C. Dou: Adv. Mater. 29 (2017). https://doi.org/10.1002/adma.201604528

16 Y. Engel, R. Elnathan, A. Pevzner, G. Davidi, E. Flaxer, and F. Patolsky: Angew Chem. Int. Ed. 49 (2010) 6830. https://doi.org/10.1002/anie.201000847

17 A. P. Cao, W. Zhu, J. Shang, J. H. Klootwijk, E. J. R. Sudholter, J. Huskens, and L. C. P. M. de Smet: Nano Lett 17 (2017) 1. https://doi.org/10.1021/acs.nanolett.6b02360

18 Z. Yang, X. C. Dou, S. L. Zhang, L. J. Guo, B. Y. Zu, Z. F. Wu, and H. B. Zeng: Adv. Funct. Mater. 25 (2015) 4039. https://doi.org/10.1002/adfm

19 D. L. Wang, H. S. Sun, A. T. Chen, S. H. Jang, A. K. Y. Jen, and A. Szep: Nanoscale 4 (2012) 2628. https://doi. org $/ 10.1039 / \mathrm{c} 2 \mathrm{nr} 30107 \mathrm{e}$

20 J. A. Howarter and J. P. Youngblood: Langmuir 22 (2006) 11142. https://doi.org/10.1021/la061240g

21 R. M. Pasternack, S. R. Amy, and Y. J. Chabal: Langmuir 24 (2008) 12963. https://doi.org/10.1021/la8024827

22 X. Yang, A. R. Gao, Y. L. Wang, and T. Li: Nano Res. 11 (2018) 1520. https://doi.org/10.1007/s12274-017$1768-\mathrm{z}$

23 C. F. Bernasconi: J. Org. Chem. 36 (1971) 1671. https://doi.org/ 10.1021/jo00811a022

24 D. R. Shankaran, T. Kawaguchi, S. J. Kim, K. Matsumoto, K. Toko, and N. Miura: Anal. Bioanal. Chem. 386 (2006) 1313. https://doi.org/10.1007/s00216-006-0699-4

25 A. Gao, X. Yang, J. Tong, L. Zhou, Y. Wang, Z. Jianlong, H.-J. Mao, and T. Li: Biosens. Bioelectron. 91 (2017) 482.

26 N. Lu, A. R. Gao, P. F. Dai, H. J. Mao, X. L. Zuo, C. H. Fan, Y. L. Wang, and T. Li: Anal. Chem. 87 (2015) 11203. https://doi.org/10.1021/acs.analchem.5b01729 


\section{About the Authors}

Xingqi Liu received her B.S. degree from the Institute of NBC Defence, Beijing, in 2016. Presently, she is a joint Master student in Shanghai Institute of Microsystem and Information Technology, Chinese Academy of Sciences. Her research interests are biochemical sensing materials and gas sensors.

Shixing Chen received his B.S. degree from Dalian Medical University in 2011 and his Ph.D. degree from Shanghai Institute of Applied Physics, Chinese Academy of Sciences, in 2016. He is currently an assistant Research Fellow at the Shanghai Institute of Microsystem and Information Technology, Chinese Academy of Sciences. His research interests focus on nanomaterials for electrochemical and bioanalytical applications, and biosensor construction.

Hui Wang received his B.S. degree from Jilin University in 2012. Presently, he is a Ph.D. student in Shanghai Institute of Microsystem and Information Technology, Chinese Academy of Sciences. His research interests include the fabrication and application of micro-nano fused sensors.

Anran Gao received her B.S. degree from Qingdao University in 2008 and her Ph.D. degree from Shanghai Institute of Microsystem and Information Technology, Chinese Academy of Sciences, in 2013. Her research interests include the design, analysis, and fabrication of semiconductor devices and their applications in biochemical sensing.

Yuelin Wang received his B.S. degree from Zhejiang University in 1982, his M.S. degree from Harbin Institute of Technology in 1985, and his Ph.D. degree from Tsinghua University in 1989. $\mathrm{He}$ is currently a professor at Shanghai Institute of Microsystem and Information Technology. His research interests include microelecromechanical systems and nanoelectromechanical systems.

Tie Li received his B.S. and Ph.D. degrees in condensed matter physics from the University of Science and Technology of China, Hefei, in 1992 and 1997, respectively. He is currently a professor in the MEMS Lab at Shanghai Institute of Microsystem and Information Technology. His research interests include the mechanism, fabrication, and application of micro-nano fused sensors.

Hongpeng Zhang received his B.S. degree from Jilin University in 1998 and his Ph.D. degree from the College of Chemistry and Molecular Engineering, Peking University, in 2012. His research interests include the design, preparation, and characterization of catalysts and adsorbents. 
Zhiping Huang received his M.S. degree from the Institute of NBC Defence in 1999 and his Ph.D. degree from Tsinghua University in 2007. His research interests include the design and synthesis of functional materials and their applications in chemical detection.

Zhenxing Cheng received his B.S. degree from the Institute of NBC Defence, Beijing, in 1986 and his M.S. and Ph.D. degrees from University Paris VI, France, in 1988 and 1992, respectively. Since 1993, he has been a professor at the Institute of NBC Defence. His research interests are in sensor technology for chemical reconnaissance. 